\title{
Ökonomisierung der Medizin? \\ Die Einführung der DRGs an Schweizer Spitälern als ethische Herausforderung
}

Der Schweiz steht - die Nationale Ethikkommission im Bereich Humanmedizin (NEK-CNE) und die Schweizerische Akademie der Medizinischen Wissenschaften (SAMW) haben verschiedentlich darauf hingewiesen - mit der Einführung von SwissDRG (diagnosebezogene Fallpauschalen) ein Systemwechsel in der Finanzierung von Spitalleistungen bevor, der nicht nur gesundheitspolitische und ökonomische, sondern auch ethische Fragen aufwirft [1, 2]. Die einschneidende Veränderung besteht darin, dass ab 2012 Spitalleistungen mit einem gemäss gestellter Diagnose festgelegten Preis versehen und nicht die tatsächlich entstandenen Kosten übernommen werden. In jüngster Zeit ist in der Schweiz ein Boom von Veranstaltungen zur DRG-Thematik zu verzeichnen, die vor allem die gesundheitsökonomischen Vorzüge herauszustreichen versuchten und die Herausforderungen der technischen Umsetzung eines DRG-Kodierungssystems erläuterten. Die Einführung der DRGs ist aber nicht eine neutrale verwaltungstechnische Massnahme, sondern gibt Anlass zum Nachdenken über die Rolle des Spitals in der Gesellschaft, über Risiken von Einbussen einer patientenorientierten Versorgungsqualität, über Fragen der Verteilungs- und Zugangsgerechtigkeit und über den Datenschutz im Gesundheitswesen. Vor diesem Hintergrund hat sich die NEK-CNE zusammen mit der SAMW entschieden, am 10. Juni 2009 in Bern ein öffentliches Symposium mit dem Titel «Ökonomisierung der Medizin? Die Einführung der DRGs an Schweizer Spitälern als ethische Herausforderung» zu veranstalten. Die Aufmerksamkeit soll sich dabei vor allem auf die Gruppen richten, deren Spitalalltag und -erfahrung sich durch die DRG-Einführung ändern könnte: zum einen die Gruppe vulnerabler Patientinnen und Patienten (z. B. Kinder), zum anderen die betroffenen Berufsgruppen von Ärzteschaft und Pflege. Inwiefern eine Begleitforschung diese Änderungen sichtbar machen könnte, um allenfalls rechtzeitig flankierende Massnahmen zu empfehlen, wird ebenfalls Gegenstand der Debatte sein. Daraus ergeben sich für das Symposium folgende Schwerpunktthemen:

- Welche Gruppen von Patientinnen und Patienten werden in DRG-Systemen privilegiert, welche sind besonders verletzlich und wie können letztere vor negativen Auswirkungen geschützt werden?

- Welches sind die Auswirkungen einer DRGbasierten Finanzierung auf die verschiedenen Berufsgruppen und deren Bedeutung im medizinischen Alltag?

- Was muss vorgekehrt werden, damit die Umsetzung von Fürsorge- und Gerechtigkeitspostulaten in der medizinischen und pflegerischen Praxis auch nach Einführung der DRGs gewährleistet bleibt?

- Welche Problemkreise müssen begleitend zur Einführung der DRGs wissenschaftlich untersucht und beobachtet werden, um unerwünschten Entwicklungen rechtzeitig entgegenwirken zu können?

Bei der Diskussion dieser Punkte durch Expertinnen und Experten aus Praxis und Wissenschaft sowie der beteiligten Organisationen gilt ein spezielles Augenmerk dem Einbezug der Erfahrungen, die in Teilen der Schweiz (z. B. Tessin) und im angrenzenden Ausland (v. a. Deutschland) bisher mit DRG-Systemen gesammelt werden konnten. Das Symposium richtet sich an interessierte Fachkreise aus dem Gesundheitswesen und an die breite interessierte Öffentlichkeit. Im Rahmen einer Abendveranstaltung findet eine Podiumsdiskussion mit Vertreterinnen und Vertretern aus der Politik und dem Gesundheitswesen statt.

1 NEK-CNE Stellungnahme Nr. 15. Zur Einführung von diagnosebezogenen Fallpauschalen in Schweizer Spitälern. Schweiz Ärztezeitung.

2008;89(36):1533-6. www.nek-cne.ch

2 SAMW-Bulletin Nr. 1/2009, das schwerpunktmässig der Einführung der DRGs gewidmet ist.

www.samw.ch $\rightarrow$ Publikationen $\rightarrow$ SAMWbulletins $\rightarrow$ $1 / 2009$. 


\section{Programm}

\section{Symposium}

Mittwoch, 10. Juni 2009, Auditorium Ettore Rossi, Inselspital (Kinderklinik), Bern

9.30 Begrüssung und Einführung ins Symposium. Prof. Christian Kind,

9.45 Einführung in die Thematik. Oliver Peters, Directeur administratif et financier, CHUV Lausanne: Ce qu'ils sont et ce qu'ils ne sont pas: opportunités et risques des DRG à la lumière des expériences faites et des modalités de leur introduction en Suisse

10.15-12.00 Vulnerable Patientinnen und Patienten

Dr. Bernard Braun, Bremen: Der Einfluss der Einführung der DRGs auf die Situation der Patientinnen und Patienten in Deutschland

Gianni Luchessa, capo servizio amministrazione e finanze, Ente ospedaliero ticinese Locarno: Les DRG influencent-ils la situation des patients dans les institutions? L'expérience du Tessin

Dr. Michele Losa, Kinderspital St. Gallen: DRGs und die spezifischen Bedürfnisse der Kinder in pädiatrischen Einrichtungen

Dr. Bertrand Kiefer, membre de la NEK-CNE, Genève: L'introduction des DRG et la situation des patients dans une perspective éthique

13.15-15.15 Betroffene Berufsgruppen

PD Dr. Arne Manzeschke, Universität Bayreuth; Dr. Pierre-François Cuénoud, Comité central FMH, Conseil d'administration SwissDRG AG, chirurgien-chef à l'hôpital cantonal de Sion: Zum Einfluss der DRGs auf Rolle und Professionsverständnis der Ärztinnen und Ärzte Prof. Sabine Bartholomeyczik, Universität Witten/Herdecke: Zum Einfluss der DRGs auf die Aufgaben und die Rolle der Pflegenden

Barbara Gassmann, Vizepräsidentin SBK, Bern: DRGs und die Kontinuität der Pflege: Zur Arbeitsteilung zwischen stationärem und ambulantem Bereich

Dr. Samia Hurst, membre de la CCE de l'ASSM, Genève: L'introduction des DRGs et leur influence sur le personnel médical dans une perspective éthique

$15.45 \quad$ Begleitforschung

PD Dr. Simon Hölzer, Geschäftsführer SwissDRG AG, Bern: Begleitforschung als Erfolgsfaktor der Einführung der DRGs

Prof. Dr. Nikola Biller-Andorno und Dr. Verina Wild, Universität Zürich: Anforderungen an die Begleitforschung zur Einführung der DRGs aus ethischer Sicht

17.00 Ausblick. Prof. Peter Suter, Präsident SAMW, Genf: Rollen der Berufsgruppen, Aufgaben des Spitals: ein Blick in die Zukunft

17.30 Resümee und Abschluss des Symposiums. Dr. Markus Zimmermann-Acklin, Universität Luzern, Vizepräsident der ZEK der SAMW

17.45 Ende des Symposiums

19.15-20.45 Abendveranstaltung: Herausforderungen und Chancen der Einführung der DRGs an Schweizer Spitälern

\section{Podiumsdiskussion mit:}

- Regierungsrat Dr. Philippe Perrenoud, Gesundheitsdirektor des Kantons Bern (angefragt)

- Nationalrat Dr. Ignazio Cassis

- Nationalrätin Bea Heim

- Dr. Jacques de Haller, Präsident FMH

- Dr. Bernhard Wegmüller, Geschäftsführer H+ Die Spitäler der Schweiz, Verwaltungsrat SwissDRG AG

- PD Dr. Silvia Käppeli, Universität Zürich, Mitglied der NEK-CNE

Moderation: Ellinor von Kauffungen

Tagungssprachen: Deutsch und Französisch (mit Simultanübersetzung)

Tagungsbeitrag: Fr. 50.-

Anmeldung: Schweizerische Akademie der Medizinischen Wissenschaften, Generalsekretariat, Petersplatz 13, 4051 Basel, Fax 06126990 39, E-Mail: mail@samw.ch 